\title{
Dendrimer-modified gelatin methacrylate hydrogels carrying adipose-derived stromal/ stem cells promote cartilage regeneration
}

\author{
Fengyi Liu ${ }^{1,2,3 \dagger}$, Xu Wang ${ }^{1,2,3 \dagger}$, Yuzhou Li ${ }^{1,2,3}$, Mingxing Ren ${ }^{1,2,3}$, Ping He $\mathrm{e}^{1,2,3}$, Lu Wang ${ }^{1,2,3}$, Jie Xu $\mathrm{Xu}^{1,2,3^{*}}$, \\ Sheng Yang ${ }^{1,2,3^{*}}$ (D) and Ping $\mathrm{Ji}^{1,2,3^{*}}$
}

\begin{abstract}
Background: Cartilage defects pose a significant burden on medical treatment, leading to an urgent need to develop regenerative medicine approaches for cartilage repair, such as stem cell therapy. However, the direct injection of stem cells can result in insufficient delivery or inaccurate differentiation. Hence, it is necessary to choose appropriate stem cell delivery scaffolds with high biocompatibility, injectability and chondral differentiation induction ability for cartilage regeneration.
\end{abstract}

Methods: In this study, the photocrosslinked gelatin methacrylate (GelMA) hydrogel with high cell affinity and plasticity was selected and strengthened by incorporating methacrylic anhydride-modified poly(amidoamine) (PAMAMMA) to fabricate an adipose-derived stromal/stem cells (ASCs) delivery scaffold for cartilage repair. The physiochemical properties of the GeIMA/PAMAM-MA hydrogel, including the internal structure, stability and mechanical properties, were tested. Then, ASCs were encapsulated into the hydrogels to determine the in vitro and in vivo chondrogenic differentiation induction abilities of the GeIMA/PAMAM-MA hydrogel.

Results: Compared with the GelMA hydrogel, the GeIMA/PAMAM-MA hydrogel exhibited more uniform structure, stability and mechanical properties. Moreover, on the basis of good biocompatibility, the hybrid hydrogel was proven to exert a sufficient ability to promote cartilage regeneration by in vitro three-dimensional (3D) culture of rASCs and in vivo articular cartilage defect repair.

Conclusions: The injectable photocrosslinked GeIMA/PAMAM-MA hydrogel was proven to be a capable stem cell carrier for cartilage repair and provides new insight into the design strategy of stem cell delivery scaffolds.

Keywords: Cartilage regeneration, Stem cell therapy, Injectable hydrogel, GelMA, PAMAM

\section{Background}

The repair of cartilage defects caused by degenerative disease, congenital anomalies or trauma is a demanding undertaking in the clinic, as cartilage tissue lacks

\footnotetext{
*Correspondence: xujie@hospital.cqmu.edu.cn; 500283@cqmu.edu.cn; jiping@hospital.cqmu.edu.cn

${ }^{\dagger}$ Fengyi Liu and Xu Wang have contributed equally to this work

${ }^{1}$ College of Stomatology, Chongqing Medical University, Chongqing, China

Full list of author information is available at the end of the article
}

sufficient self-healing capacity $[1,2]$. The inherent physiological characteristics of cartilage tissue, including its avascular nature and high matrix-to-cell ratio, lead to the difficulties in developing a successful therapeutic schedule for cartilage regeneration $[3,4]$. Conventional surgical procedures for treating cartilage defects, including microfractures and autologous and allogeneic implantation, have certain limitations so that the shape and function of cartilage defects cannot be completely restored $[5,6]$. To overcome the difficulties original author(s) and the source, provide a link to the Creative Commons licence, and indicate if changes were made. The images or other third party material in this article are included in the article's Creative Commons licence, unless indicated otherwise in a credit line to the material. If material is not included in the article's Creative Commons licence and your intended use is not permitted by statutory regulation or exceeds the permitted use, you will need to obtain permission directly from the copyright holder. To view a copy of this licence, visit http://creativecommons.org/licenses/by/4.0/. The Creative Commons Public Domain Dedication waiver (http://creativeco mmons.org/publicdomain/zero/1.0/) applies to the data made available in this article, unless otherwise stated in a credit line to the data. 
in treatment, stem cell therapy has been developed to provide promising alternative strategies for regenerating cartilage $[7,8]$.

Among the various kinds of stem cells, adipose-derived stromal/stem cells (ASCs) are considered highly promising in regenerative medicine because of their abundance, ready accessibility with minimally invasive procedures and anti-inflammatory properties [9-11]. However, the direct injection of stem cells has some shortcomings, including excessive cell dosage, induced cell damage or uncontrolled stem cell differentiation [12-14]. Therefore, the delivery of ASCs into the targeted cartilage defect area needs to be carried out in a scaffold-guided manner. In addition, the direction of ASCs differentiation could be controlled by the composition, architecture, physicochemical and mechanical properties of cell delivery vehicles $[9,15,16]$. Accordingly, it is important to select cell delivery scaffolds with appropriate properties for the chondrogenic differentiation induction of ASCs.

As tissue engineering scaffolds, hydrogels have received substantial amounts of attention due to their good biocompatibility, tunable physicochemical properties and structure similar to the three-dimensional (3D) crosslinked hydrophilic network of biological tissues.[17, $18]$. With these bionic properties, hydrogels are better able to benefit the localization, attachment, proliferation, and desired differentiation of stem cells [19]. To achieve adequate repair of cartilage defects, hydrogels require injectability and in situ gelation, such as the widely used synthetic strategy of photocrosslinking [20-22]. GelMA hydrogels are among the most commonly used photocrosslinked hydrogels with high biocompatibility to mimic the native extracellular environment [23, 24]. However, the potential applications of GelMA hydrogels are hindered due to their low mechanical properties and excessive degradation speed, which prevents the material from utility in the regeneration of load-bearing tissues such as cartilage $[25,26]$. Therefore, it is essential to combine GelMA with other materials to ameliorate these limitations.

Poly(amidoamine) (PAMAM) dendrimers are spherical hyperbranched polymers with specific controllable sizes and easily modified surfaces [27, 28]. Owing to these outstanding properties, PAMAM dendrimers have long been used in the fields of biomedicine such as drug delivery, molecular imaging and gene therapy [29-31]. Moreover, PAMAM dendrimer incorporated materials have shown promising results when investigated in skin and corneal tissue engineering applications [32, 33]. In addition, we have previously introduced PAMAM dendrimer into the hybrid poly(lactic acid)-b-poly(ethylene glycol)b-poly(lactic acid) (PEG-LA-DA) hydrogel system for bone tissue engineering [34]. Hence, PAMAM has great potential for the building composite tissue engineering scaffolds for cartilage regeneration.

In this research, GelMA and methacrylic anhydride (MA)-modified PAMAM (PAMAM-MA) were used to fabricate an injectable stem cell-laden photocrosslinked hydrogel for cartilage regeneration (Fig. 1). GelMA hydrogels constituted the control group. To analyze the effect of the composition of hydrogels on their physiochemical properties, we compared the structure, stability and mechanical strength of two groups of GelMA/ PAMAM-MA hydrogels through various tests. We then performed a series of in vitro and in vivo studies to verify whether the addition of PAMAM-MA can induce the chondrogenic differentiation of stem cells and repair cartilage defects properly. ASCs were encapsulated in two groups of hydrogels for in vitro stem cell chondrogenic differentiation analysis by detecting the expression of chondrogenic-related genes and proteins. For in vivo cartilage defects, we constructed a knee articular cartilage defect model for further therapy. Two groups of injectable stem cell-laden hydrogels were used for in situ treatment to analyze their cartilage defect repair abilities.

\section{Methods}

\section{Synthesis of GeIMA and PAMAM-MA}

The synthesis of GelMA and PAMAM-MA followed protocols described previously [26, 35]. Gelatin powder from cold-water fish skin (Sigma-Aldrich, USA) was dissolved in phosphate-buffered saline (PBS; HyClone, USA) at a concentration of $100 \mathrm{mg} / \mathrm{mL}$ at $50{ }^{\circ} \mathrm{C}$. Methacrylic anhydride (MA, Aladdin, China) was added to the gelatin solution at a rate of $0.5 \mathrm{~mL} / \mathrm{min}(20 \%, \mathrm{w} / \mathrm{v})$. The mixture was reacted for $3 \mathrm{~h}$ under stirring conditions at $50{ }^{\circ} \mathrm{C}$. The reaction was then terminated by the addition of excessively warm PBS. The GelMA solution was dialyzed against deionized water for 7 days at $40{ }^{\circ} \mathrm{C}$ to remove excess residual small molecules, and then the GelMA solution was filtered, freeze-dried and stored at $-20{ }^{\circ} \mathrm{C}$. PAMAM (Weihai CY Dendrimer Technology Co., Ltd., China) was modified with MA in accordance with the same protocol above to synthesize PAMAM-MA.

\section{Fourier transform infrared (FTIR) spectroscopy analysis}

FTIR spectroscopy was performed to verify whether GelMA and PAMAM-MA were synthesized successfully. Gelatin, PAMAM, GelMA and PAMAM-MA were tested to compare the composition of two targeted polymers before and after their modification with MA. To obtain the FTIR spectra, FTIR analysis was performed via an FITR spectrometer (Nicolet iS50, Thermo Fisher Scientific, USA) by recording 256 scans, each with a spectral width ranging from 500 to $4000 / \mathrm{cm}$ at a spectral resolution of $4 / \mathrm{cm}$. 


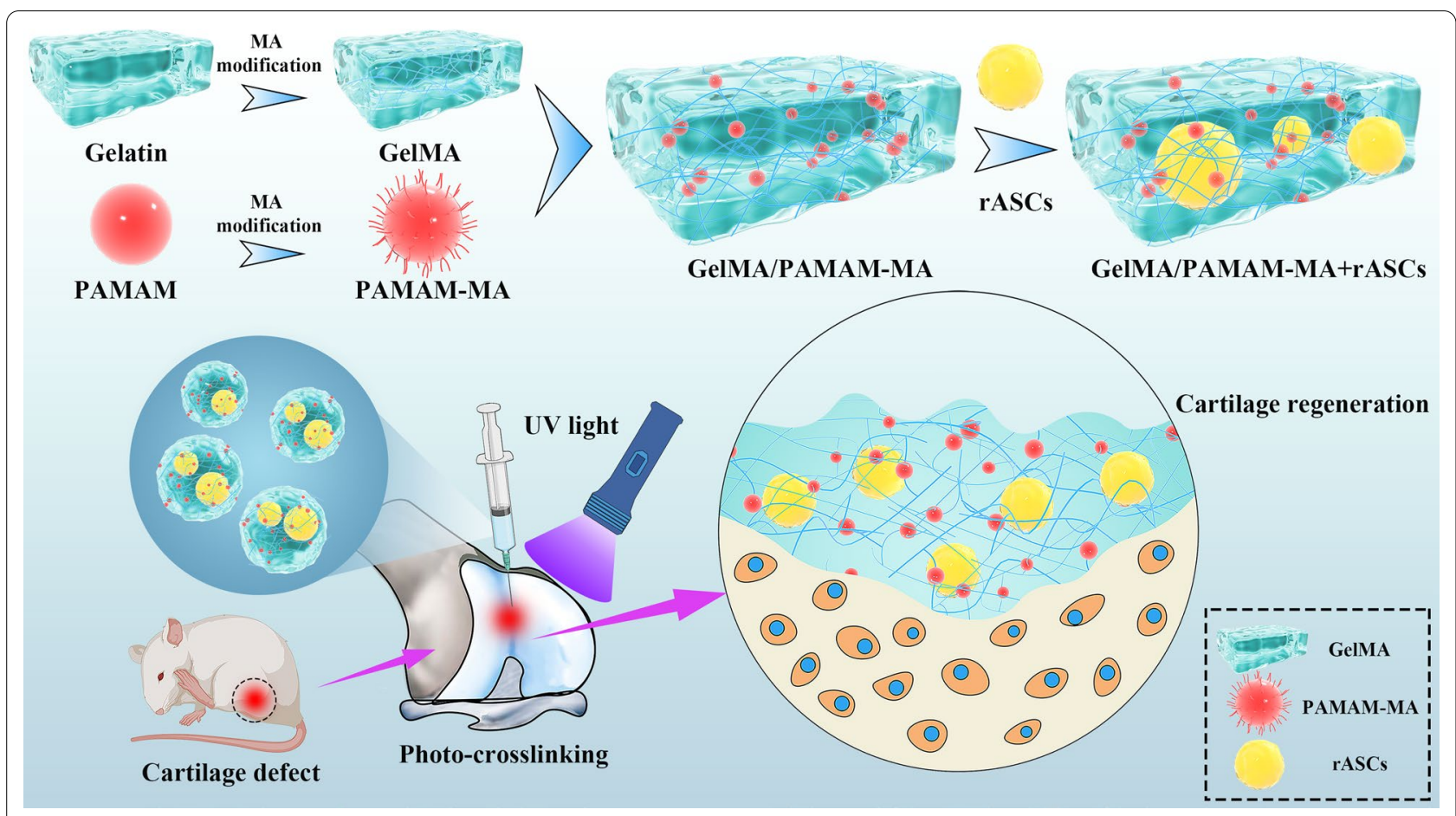

Fig. 1 Schematic diagram of the injectable stem cell-laden photocrosslinked GeIMA/PAMAM-MA hydrogel for cartilage regeneration

\section{Formation of GeIMA/PAMAM-MA hydrogels}

Lyophilized GelMA and PAMAM-MA were dissolved in photoinitiator solution separately at a concentration of $100 \mathrm{mg} / \mathrm{mL}$. To prepare the photoinitiator solution, 2-hydroxy-1(4-(hydroxyethoxy)pheny)-2-methyl-1-propanone (Irgacure 2959, Sigma, USA) was dissolved in PBS at a concentration of $2 \mathrm{mg} / \mathrm{mL}$ under ultrasonic vibration at $50{ }^{\circ} \mathrm{C}$. GelMA and PAMAM-MA solutions were then mixed to prepare the prepolymer solutions. The final concentration of GelMA in prepolymer solutions was $50 \mathrm{mg} / \mathrm{mL}$, and PAMAM-MA solutions were prepared at the final concentrations of 0 or $20 \mathrm{mg} / \mathrm{mL}$. The prepolymer solutions were exposed to $365 \mathrm{~nm}$ ultraviolet (UV) light for $30 \mathrm{~s}$ to synthesize hydrogels, after which they were soaked in PBS for further use.

\section{Hydrogel pore sizes and internal structure test}

The effect of the composition of hydrogels on the pore size and network structure of hydrogels was characterized by scanning electron microscopy (SEM) and frozen sectioning. GelMA/PAMAM-MA hydrogels were frozen at $-80{ }^{\circ} \mathrm{C}$ and then lyophilized before being sputter coated with $\mathrm{Au}-\mathrm{Pd}(2 \mathrm{~nm})$ and examined via SEM (Hitachi-SU8010, Japan). For frozen section detection, two groups of hydrogels were immersed in PBS for $48 \mathrm{~h}$, embedded in optimal cutting temperature compound (OCT, Sakura Finetek, USA), and then frozen at $-80{ }^{\circ} \mathrm{C}$ overnight. The hydrogels were sliced into $10 \mu \mathrm{m}$-thick sections by using a cryostat (Leica, Germany) and then stained with hematoxylin (Solarbio, China). The pore sizes of the hydrogels were measured with ImageJ software (NIH, USA).

\section{Swelling ratio analysis}

The swelling ratio of GelMA/PAMAM-MA hydrogels was determined by measuring the weight change in the hydrogel before and after dehydration to analyze the effect of hydrogel components on their stabilities. Hydrogels were incubated in PBS at room temperature for $48 \mathrm{~h}$, and the initial weight $(\mathrm{Wi})$ was weighed. The hydrogels were then freeze dried for $24 \mathrm{~h}$, and the dry weight (Wd) was measured. The following equation was used to calculate the swelling ratio: swelling ratio $=(\mathrm{Wi}-\mathrm{Wd}) /$ Wd $\times 100 \%$.

\section{In vitro degradation tests}

The degradation of GelMA and GelMA/PAMAM-MA hydrogels was performed in vitro by immersing hydrogels into PBS solution containing $1 \mathrm{unit} / \mathrm{mL}$ collagenase type II (Solarbio, China). The initial weight of the lyophilized hydrogels $(n=4)$ was measured and recorded, and hydrogels were immersed in $1 \mathrm{~mL}$ of degradation solution and incubated under shaking at $37^{\circ} \mathrm{C}$. The degraded hydrogels were washed twice and lyophilized at each set 
time point (after 12 h, 1 day, 2 days, 4 days and 7 days). The weight of the freeze-dried hydrogels was measured. The degradation solution was replaced by freshly prepared degradation solution.

\section{Mechanical test}

The compressive strength of two groups of GelMA/ PAMAM-MA hydrogels was measured to analyze the effect of hydrogel composition on the mechanical properties of hydrogels. The compressive modulus of GelMA and GelMA/PAMAM-MA hydrogel disks (thickness $=2 \mathrm{~mm}$, diameter $=5 \mathrm{~mm}$ ) was determined by using a universal testing machine (MSD, USA). The compressive stress-strain curves were obtained at a strain rate of $0.2 \mathrm{~mm} / \mathrm{min}$. The compressive modulus was determined by taking the slope of the linear region of the stressstrain curves at $0-20 \%$ strain.

\section{Isolation and identification of rat adipose-derived stromal/ stem cells}

Rat adipose-derived stromal/stem cells (rASCs) were isolated and identified as described previously [36]. Briefly, rat adipose tissues of the groin were isolated and washed three times with PBS containing 1\% penicillin-streptomycin (HyClone, USA). Adipose tissues were then cut and digested with type I collagenase (Sigma, USA), after which they were dissolved in Dulbecco's modified Eagle's medium (DMEM; Gibco, USA) for $1 \mathrm{~h}$ at $37^{\circ} \mathrm{C}$. After centrifugation at $1000 \mathrm{rpm}$ for $5 \mathrm{~min}$, the cell pellet was suspended DMEM that included 10\% fetal bovine serum (HyClone, Australia) and 1\% penicillin-streptomycin. The cell suspension was filtered through a $70 \mu \mathrm{m}$ cell filter and centrifuged for another $5 \mathrm{~min}$. Cell pellets were suspended in growth medium and incubated in a $\mathrm{CO}_{2}$ incubator at $37^{\circ} \mathrm{C}$.

Third generation rASCs were used for identification. Flow cytometry (FCM) was used to detect the expression of surface antibodies related to stem cell identification, including CD90, CD44, CD45 and CD31 (BioLegend, USA). Cells were digested and centrifuged at $1000 \mathrm{rpm}$ and washed with PBS. After incubation with antibodies $(1: 100)$ at $4{ }^{\circ} \mathrm{C}$ for $30 \mathrm{~min}$, the cells were subjected to FCM (Becton Dickinson, USA). The results were analyzed with FlowJo software (FlowJo, USA). rASCs were also identified by osteogenic, adipogenic and chondrogenic differentiation. Third-generation rASCs were induced in osteogenic differentiation medium (Cyagen, China) for 7 days and stained with an alkaline phosphatase staining kit (Beyotime Biotechnology, China) to verify the osteogenic differentiation of stem cells. rASCs were induced in adipogenic differentiation medium (Cyagen, China) for 21 days and stained with an oil red O staining kit (Solarbio, China) to verify adipogenesis. For chondrogenic differentiation, rASCs were induced in chondrogenic differentiation medium (Cyagen, China) for 14 days. Cell pellets were embedded in OCT for frozen sectioning. After storage in $\mathrm{a}-80^{\circ} \mathrm{C}$ refrigerator overnight, cell pellets were cut into $10 \mu \mathrm{m}$ sections and stained with Alcian Blue solution. The staining results were observed and imaged by inverted microscopy (Olympus, Japan).

\section{In vitro 3D cell culture}

For 3D cell culture, rASCs were resuspended in two groups of GelMA/PAMAM-MA prepolymer solutions at a density of $1 \times 10^{7}$ cells $/ \mathrm{mL}$. The mixture was added to polydimethylsiloxane (PDMS) molds and exposed to $365 \mathrm{~nm}$ UV light for $30 \mathrm{~s}$. The synthesized hydrogels were immersed in growth medium and cultured in a $\mathrm{CO}_{2}$ incubator at $37^{\circ} \mathrm{C}$.

\section{Live/dead cell staining}

To assess the cell viability of rASCs encapsulated in GelMA/PAMAM-MA hydrogels, live/dead cell staining was performed. After rASCs were encapsulated in two groups of GelMA/PAMAM-MA hydrogels for 1 day, 4 days and 7 days, the hydrogels were washed with PBS twice and stained with calcein-AM/PI dyeing solution (Bestbio, China) for $20 \mathrm{~min}$. Subsequently, the hydrogels were washed with PBS three times and observed by confocal microscopy (CLSM, Zeiss LSM 9100, Germany). ImageJ software was used to count and analyze the numbers of living cells and dead cells.

\section{Cytoskeleton/nucleus fluorescent staining}

To evaluate the cell spreading status, rASCs in GelMA/ PAMAM-MA hydrogels were measured with cytoskeleton/nucleus fluorescent staining after 1, 4 and 7 days of cell culture. Alexa Fluor 488-conjugated phalloidin (Invitrogen, USA) and 4',6-diamidino-2-phenylindole (DAPI; Invitrogen, USA) were used for cytoskeletal and nuclear staining, respectively. The samples were fixed in $4 \%$ paraformaldehyde (PFA; Sigma, USA) for $10 \mathrm{~min}$ and washed with PBS three times. The hydrogels were incubated in phalloidin solution (1:300 dilution) for $2 \mathrm{~h}$ at room temperature. For DAPI/cell nuclei staining, DAPI solution (1:1000 dilution) was used to incubate hydrogels for $30 \mathrm{~min}$. The hydrogels were then measured by confocal microscopy to observe the extent of cell spreading.

\section{Quantitative real-time polymerase chain reaction (qPCR) analysis}

qPCR was performed to quantitatively evaluate the messenger ribonucleic acid (mRNA) expression level of chondrogenic differentiation genes in rASCs encapsulated in two groups of hydrogels, including sex determining region Y-box transcription factor 9 (SOX9), 
aggrecan (ACAN), type II collagen (Col-II) and type $\mathrm{X}$ collagen (Col-X). rASCs were encapsulated in hydrogels and cultured in chondrogenic induction medium (Cyagen, China) for 7 days. Total RNA was extracted and examined by a NanoDrop spectrophotometer (Thermo Fisher Scientific, USA) to determine the concentration. A PrimeScript RT Master Mix Kit (Takara, Japan) was used to reverse-transcribe RNA to complementary DNA (cDNA) according to the manufacturer's instructions. The cDNA of target genes was mixed with SYBR Green PCR Master Mix (Takara, Japan) and loaded into a Real-Time PCR System (Bio-Rad, USA) to analyze the expression levels of chondrogenesis-related genes; glyceraldehyde-3-phosphate dehydrogenase (GAPDH) was used as the housekeeping gene. The sense and antisense primer sequences of the above genes are listed in Additional file 1: Supplementary information, Table S1. The relative gene expression levels were analyzed using the comparative $\mathrm{Ct}(\Delta \Delta \mathrm{Ct})$ method.

\section{Safranin-O staining}

To analyze the effect of hydrogel components on the deposition of cartilage-specific extracellular matrix (ECM) in hydrogels, safranin-O staining was used for detection. After culturing in chondrogenic induction medium for 14 days, two groups of GelMA/PAMAM-MA hydrogels were rinsed with PBS, fixed in 4\% PFA for $10 \mathrm{~min}$ and washed with PBS three times. Hydrogels were then embedded within $\mathrm{OCT}$ and stored in a $-80^{\circ} \mathrm{C}$ refrigerator overnight prior to frozen sectioning. The hydrogels were sectioned into $10 \mu \mathrm{m}$-thick sections and stained with safranin-O dyeing solution (Solarbio, China) by following the manufacturer's instructions. The staining results were recorded via inverted microscopy.

\section{Immunofluorescence (IF) staining}

IF staining was used to study the expression of the chondrogenic-associated protein SOX9 in rASCs. After culturing in chondrogenic induction medium for 21 days, cell-laden hydrogels were fixed in 4\% PFA for $10 \mathrm{~min}$ and washed with PBS three times. Hydrogels were blocked with $5 \%$ donkey serum and incubated with primary antibodies (SOX9, Abcam, USA, ab185966, 1:300) overnight at $4{ }^{\circ} \mathrm{C}$. Afterward, the hydrogels were incubated with secondary antibodies (Alexa Fluor 488-AffiniPure Donkey anti-rabbit IgG, Invitrogen, USA, \#a21206, 1:300) for $1 \mathrm{~h}$ at room temperature. Finally, the hydrogels were stained with DAPI solution and measured via confocal microscopy.

\section{In vivo rat cartilage defect repair study}

In this study, 6-week-old female Sprague Dawley rats (200-250 g weight, Chongqing, China) were used and treated with standard surgical procedures, which were conducted in accordance with protocols approved by the Ethics Committee of the Stomatological Hospital of Chongqing Medical University. Rats were anesthetized with isoflurane and defects $(1.5 \mathrm{~mm}$ in diameter, $1.0 \mathrm{~mm}$ in depth) were created by a dental drill on the surface of rat knee joints. Hydrogel prepolymer mixture solutions loaded with rASCs were injected into defects and exposed to $365 \mathrm{~nm}$ UV light for $30 \mathrm{~s}$. Four groups were established a normal control group (untreated group), blank control group (defects unrepaired group), GelMA + rASCs group and GelMA/PAMAM-MA + rASCs group.

\section{In situ observation of implanted rASCs}

To trace the rASCs implanted into the cartilage defects, we used green fluorescent protein (GFP)-labeled adenovirus (GeneChem, China) to transfect the cells. The transfected rASCs were encapsulated into GelMA/ PAMAM-MA hydrogel and injected into the defect areas. At 1 week, 2 weeks and 4 weeks after implantation, the rats were sacrificed and the cartilage defect areas were median sectioned and stained with DAPI solution to observe the rASCs in the defect areas.

\section{Histological analysis of repaired cartilage defect tissues}

At 8 weeks after repair, the rats were sacrificed. Rat knee joints were separated, washed with PBS and imaged. Samples were then fixed in $4 \%$ PFA solution for $24 \mathrm{~h}$ at $4{ }^{\circ} \mathrm{C}$. All samples were decalcified by $20 \%$ ethylenediaminetetraacetic acid (EDTA) solution for 2 months, dehydrated, embedded in paraffin, and sectioned into $5 \mu \mathrm{m}$-thick sections. To evaluate the extent of tissue reconstruction, sections of all groups were stained with hematoxylin and eosin (H\&E) staining solutions (Solarbio, China) and a safranin-O/fast green staining kit (Solarbio, China). Histological points of the repaired cartilage defect tissues were scored by 3 independent blinded observers using the International Cartilage Repair Society (ICRS) visual histological assessment [37].

\section{Immunohistochemical (IHC) staining}

For IHC staining, sections were deparaffinized and digested with pepsin for $20 \mathrm{~min}$ at $37{ }^{\circ} \mathrm{C}$ for antigen retrieval. The sections were then incubated in 3\% hydrogen peroxide solution for $10 \mathrm{~min}$ to eliminate endogenous peroxidase activity at room temperature. The sections were blocked with 5\% goat serum and incubated with proper dilutions of primary antibodies overnight at $4{ }^{\circ} \mathrm{C}$. The following primary antibodies were used: SOX9 (Abcam, USA, ab185966, 1:300) and Col-II (Santa Cruz, USA, sc-52658, 1:300). Afterward, the sections were incubated with secondary antibodies (Bioss antibodies, China: goat anti-rabbit IgG H\&L antibody, \#bs-0295G, 
1:300; goat anti-mouse IgG H\&L antibody, \#bs-0296G, $1: 300)$ for $60 \mathrm{~min}$ at room temperature. The sections were then stained with the chromogenic agent 3,3'-diaminobenzidine (DAB) to visualize the specific expression of targeted proteins. Finally, the sections were stained with hematoxylin for cell nuclear localization.

\section{Statistical analysis}

All experiments were repeated three times to verify the results. Data analysis was performed via GraphPad Prism 7.0 (GraphPad Software, USA). Statistical analysis was evaluated using Student's t test for two-group comparisons and using one-way ANOVA with Tukey's post hoc test for multigroup comparisons. A value of $p<0.05$ was considered statistically significant. The results were presented as the mean \pm Standard Deviation (SD).

\section{Results}

\section{FTIR characterization}

The synthesis of GelMA and PAMAM-MA is described in Fig. 2A. Gelatin and PAMAM were modified by MA at $50{ }^{\circ} \mathrm{C}$. FTIR was performed to verify the MA modification of gelatin and PAMAM (Fig. 2B). The intensities of the typical amide bonds at $1633 / \mathrm{cm}$ and $1538 / \mathrm{cm}$ in the spectra of gelatin and GelMA varied. Moreover, the stretching vibration peak of the $\mathrm{N}-\mathrm{H}$ group was observed at $3297 / \mathrm{cm}$, and the peak at $2941 / \mathrm{cm}$ was a C-H stretching peak. For the spectra of PAMAM and PAMAM-MA, a change in the peak at $1633 / \mathrm{cm}$ was observed for the typical amide bands and the peak at $2819 / \mathrm{cm}$ was attributed to $\mathrm{C}-\mathrm{H}$ stretching. Taken together, these changes were caused by the modification of amino groups by MA to form new amide bonds, and were evidence of successful modification of gelatin and PAMAM with MA.

\section{Synthesis and internal structure measurement of GelMA/ PAMAM-MA hydrogels}

As shown in Fig. 2A and C, the GelMA/PAMAM-MA hydrogel was synthesized after exposure to $365 \mathrm{~nm}$ UV light for $30 \mathrm{~s}$. We examined the pore sizes and internal structures of two groups of the two hydrogels under two different dry and wet conditions through SEM (Fig. 2D) and frozen section inspections (Additional file 1: Supplementary information, Fig. S1). The internal cross-linked network of the GelMA hydrogel was irregular and dispersed in both the freeze-dried state and the wet state. Correspondingly, the GelMA/PAMAM-MA hydrogel exhibited a well-distributed and integrated internal network. The pore sizes of the two groups of hydrogels were analyzed and compared, while GelMA/PAMAM-MA hydrogels presented smaller average pore sizes and more compact and uniform structures.

\section{Stability of GeIMA/PAMAM-MA hydrogels}

The swelling ratios were tested to evaluate the stability of the injectable photocrosslinked hydrogels. By measuring the wet and dry weights of hydrogels, we calculated and analyzed the swelling ratios. The results in Fig. 2E demonstrate that the swelling ratio of the hydrogels declined significantly with the addition of PAMAM-MA, which could be attributed to changes in the pore sizes of the hydrogels. Furthermore, the results of in vitro degradation tests of the two groups of hydrogels showed that the GelMA hydrogel completely degraded within $24 \mathrm{~h}$, while the GelMA/ PAMAM-MA hydrogel could retain approximately $20 \%$ of the initial mass when it degraded for 7 days (Fig. 2F). These results indicated that PAMAMMA can effectively improve the stability of hydrogels.

\section{Mechanical properties of GeIMA/PAMAM-MA hydrogels}

Mechanical properties of hydrogels profoundly regulate the differentiation of stem cells. Therefore, we introduced PAMAM-MA into the GelMA hydrogel, aiming at adjusting the mechanical properties of hydrogels to regulate the differentiation of rASCs. The mechanical properties of the two groups of GelMA/PAMAM-MA hydrogels were determined by measuring the compression modulus. We tested the compressive strength of hydrogels by using a universal testing machine. As shown in Fig. 2G, statistical analysis revealed that the compression modulus of the GelMA/PAMAM-MA hydrogel $(8.06 \pm 1.39 \mathrm{kPa})$ increased significantly with the addition of PAMAM-MA compared with that of the GelMA hydrogel $(2.56 \pm 0.26 \mathrm{kPa})$.

\section{Identification of rASCs}

The FCM analysis results in Fig. 3A revealed that rASCs were positive for the expression of mesenchymal stem cell-related surface antigens, including CD90 (99.0\%) and CD44 (97.6\%). In addition, rASCs were negative for the expression of the endothelial-related marker CD31 $(0.16 \%)$ or hematopoietic-related marker CD45 (0.11\%). The multidifferentiation ability of rASCs is shown in Fig. 3B. The positive ALP staining results revealed the osteogenic differentiation ability of rASCs after osteogenic induction culture. Oil red $\mathrm{O}$ staining also revealed visible accumulation of intracytoplasmic lipid droplets, which proved the adipogenic differentiation ability of rASCs. Moreover, Alcian blue staining of rASCs proved their chondrogenic differentiation ability. 


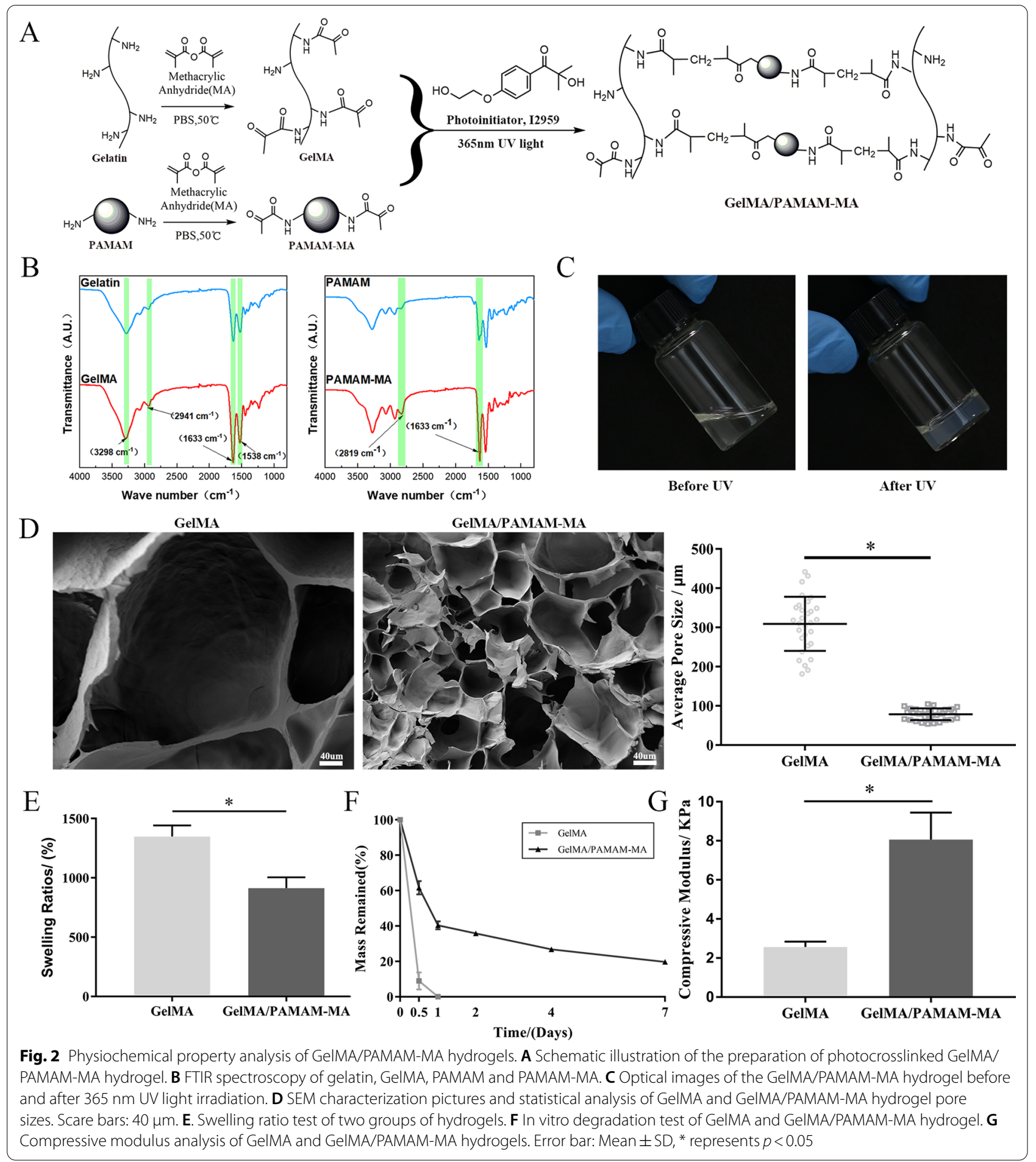

\section{In vitro biocompatibility tests}

To evaluate the biocompatibility of the two groups of hydrogels, we measured the cell viabilities of rASCs encapsulated in GelMA and GelMA/PAMAM-MA hydrogels by staining with calcein-AM/PI dyeing solution and observed them via confocal microscopy. Live/dead cell staining images of ASCs encapsulated in hydrogels after culture for 1,4 and 7 days displayed a high rate of cell viability and uniform distribution (Fig. 3C). We counted and analyzed the living and dead cell numbers, 


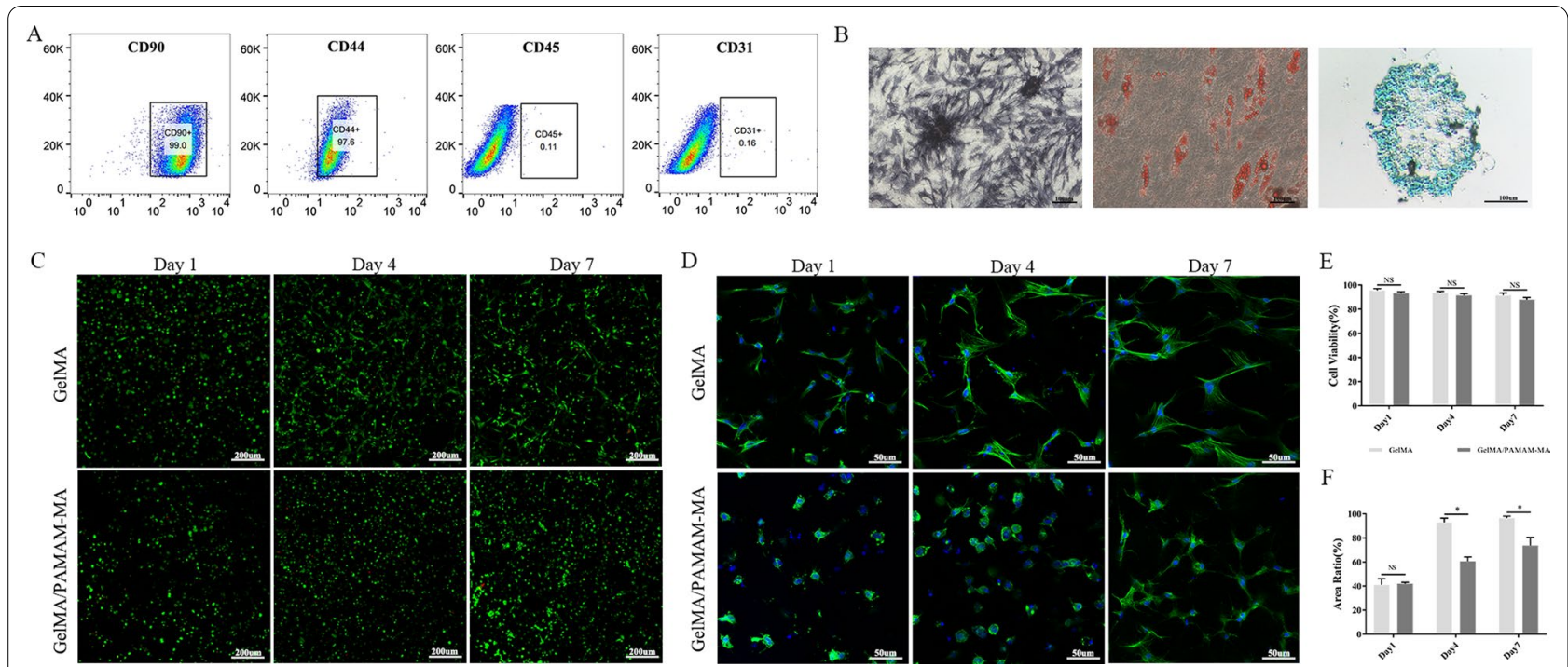

Fig. 3 Identification of rASCs and in vitro biocompatibility evaluations. A FCM analysis of the expression of stem cell identification-related antibodies in rASCs. B Representative images of the multidifferentiation ability test of rASCs. Left, ALP staining, middle, Oil red O staining, right, Alcian blue staining. Scare bars: $100 \mu \mathrm{m}$. C Representative images of live/dead rASCs cultured in GelMA and GelMA/PAMAM-MA hydrogels on days 1, 4 and 7. Green fluorescence indicates live cells, and red fluorescence indicates dead cells. Scare bars: $200 \mu m$. D Representative images of phalloidin/DAPI fluorescence images of rASCs cultured in two groups of GeIMA/PAMAM-MA hydrogels after 1 day, 4 days and 7 days. Green fluorescence indicates the cytoskeleton, and blue fluorescence indicates the nucleus. Scare bars: $50 \mu \mathrm{m}$. E Quantification of the live and dead cells. F Quantification of the cell spreading areas of rASCs cultured in two groups of hydrogels. Error bar: Mean \pm SD, ${ }^{*}$ represents $p<0.05$

and the results showed that cell viabilities of rASCs encapsulated in the two groups of hydrogels remained above $80 \%$ at all time points, with no significant differences (Fig. 3E).

We examined the spreading of rASCs in GelMA and GelMA/P AMAM-MA hydrogels by cytoskeleton/nuclei fluorescent staining at day 1 , day 4 and day 7. rASCs encapsulated in hydrogels gradually spread as the culture time increased (Fig. 3D). When comparing the spread of rASCs between the two groups of hydrogels, we found that the extent of cell spreading decreased in the GelMA/ PAMAM-MA hydrogels. The cell spreading areas of rASCs were then quantified using Image-Pro Plus software, and statistically significant decreases were observed in GelMA/PAMAM-MA hydrogels at day 4 and day 7 (Fig. 3F). rASCs in the GelMA/PAMAM-MA hydrogel presented a decreased spreading area and spherical morphology, similar to that of chondrocyte phenotype, which might be beneficial to chondrogenesis of rASCs.

\section{In vitro chondrogenesis study}

We first analyzed and compared the ability of two groups of hydrogels to regulate the chondrogenic differentiation of rASCs by detecting the expression of chondrogenic-related genes in rASCs, including the chondrogenic phenotype genes SOX9, Col-II, ACAN and Col-X. The qPCR analysis results showed varied gene expression levels of rASCs encapsulated in GelMA and GelMA/PAMAM-MA hydrogels after 1 week of in vitro differentiation (Fig. 4A). The expression of SOX9, ACAN and Col-II significantly increased in the GelMA/PAMAM-MA hydrogel, while the expression of the hypertrophic cartilage-related gene Col-X showed no significant difference between the two groups.

Furthermore, we carried out frozen sectioning and stained rASCs with safranin-O dye after 2 weeks of chondrogenic induction culture to visualize the spatial distribution of glycosaminoglycans (GAGs) in the two groups of hydrogels. Representative images showed that the deposition of GAGs increased significantly in GelMA/PAMAM-MA hydrogels compared with GelMA hydrogel (Fig. 4B). The positive stained area of GAGs was semiquantitatively analyzed by ImageJ software, and the result showed a significant difference between the two groups of hydrogels (Fig. 4D).

In addition, we performed immunofluorescence staining of SOX9 protein in rASCs encapsulated in two groups of hydrogels after 21 days of chondrogenic induction culture, and more positively stained cells could be observed in GelMA/PAMAM-MA hydrogels (Fig. 4C). The semiquantitative analysis of the immunofluorescence staining images by ImageJ software revealed a significant difference in the expression of SOX9 between the two groups (Fig. 4E). In general, our 


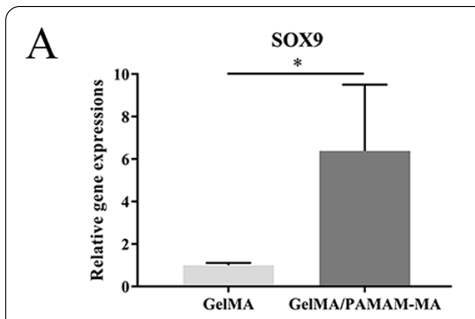

$\mathrm{B}$

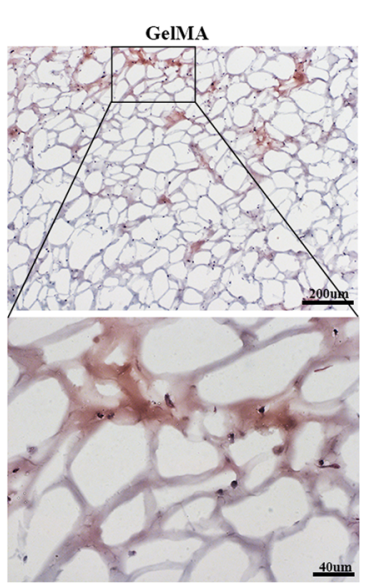

$\mathrm{D}$

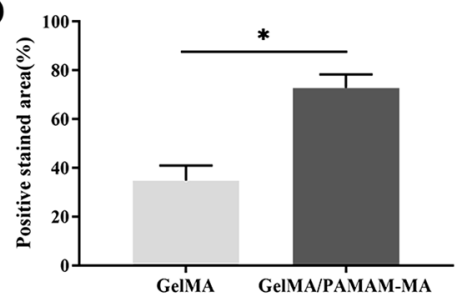

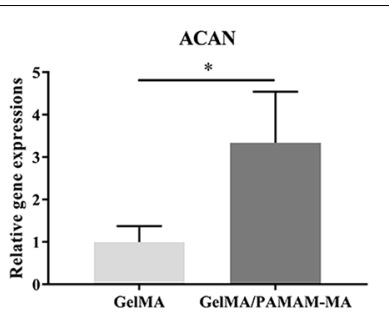
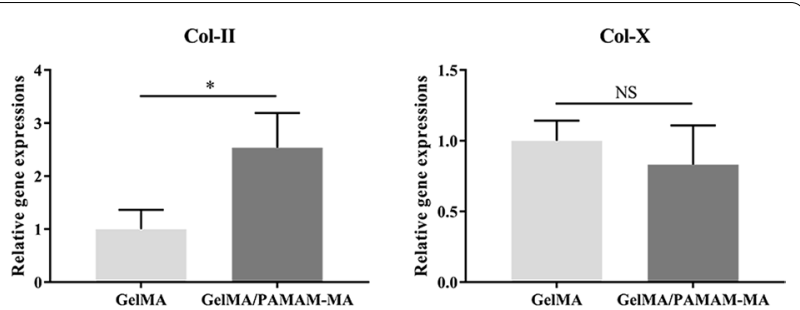

$\mathrm{C}$
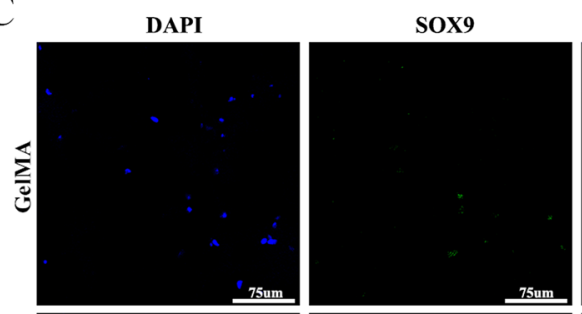

Merge
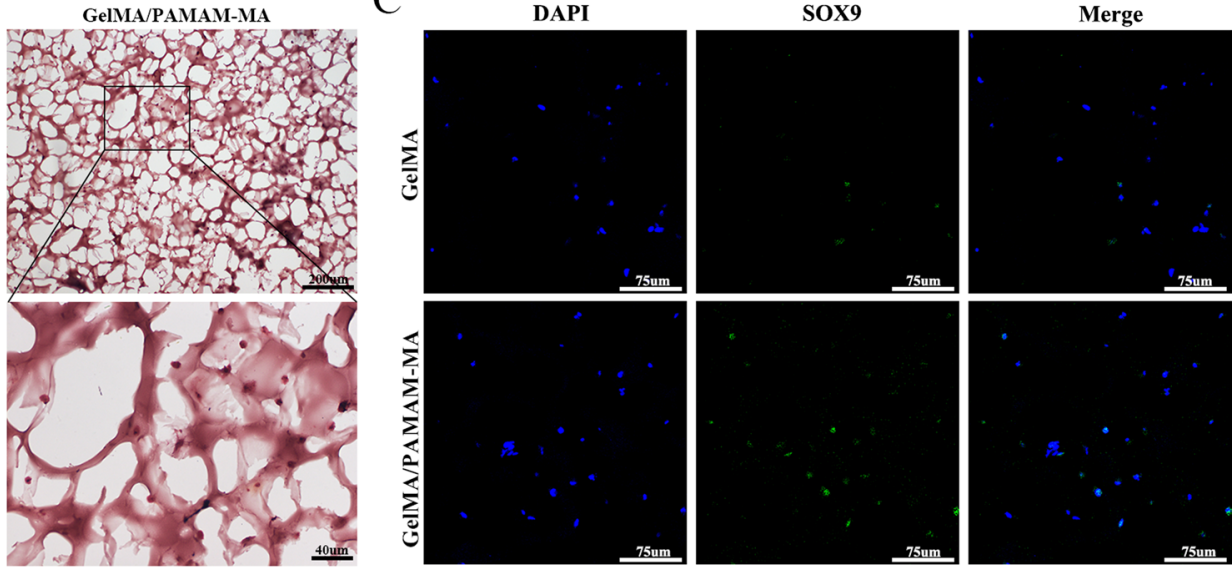

$\mathrm{E}$

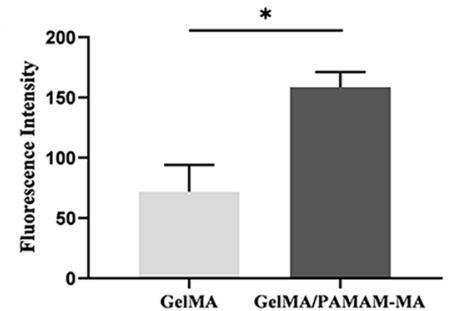

Fig. 4 In vitro chondrogenic differentiation analysis of 3D-cultured rASCs. A Quantitative real-time PCR analysis of the expression of chondrogenic differentiation-related genes in rASCs encapsulated in GelMA and GeIMA/PAMAM-MA hydrogels. B Representative images of safranin-O staining after 14 days of chondrogenic induction culture. Scale bars: above, $200 \mu \mathrm{m}$; below, $40 \mu \mathrm{m}$. C Representative images of immunofluorescence staining of SOX9 in rASCs encapsulated in two groups of hydrogels after 21 days of chondrogenic induction culture. Scale bars: $75 \mu \mathrm{m}$. D Quantification of the positively stained area of GAGs in the two groups of hydrogels. E Quantification of SOX9 expression in the two groups of hydrogels. Error bar: Mean $\pm \mathrm{SD},{ }^{*}$ represents $p<0.05$

results proved at the genetic and protein levels that the GelMA/PAMAM-MA hydrogel can better promote the chondrogenic differentiation of rASCs in vitro than can the GelMA hydrogel.

\section{In vivo cartilage repair assays}

After comparatively analyzing the ability to induce cartilage differentiation of rASCs in vitro, we tested the ability of the two groups of hydrogels to promote cartilage regeneration in vivo. Hydrogels loaded with rASCs were injected into rat knee joint articular cartilage defects for 8 weeks of cartilage repair treatment (Fig. 5A). After they were loaded into the GelMA/PAMAM-MA hydrogel, GFP-labeled adenovirus-transfected rASCs were injected into cartilage defects. These rASCs could still be observed in the cartilage defect at 1 week, 2 weeks and 4 weeks after implantation (Additional file 1: Supplementary information, Fig. S2).

Gross morphological examining, histological staining and immunohistochemical staining were performed to assess the cartilage reparative capability of the two groups of hydrogels. Representative images in Fig. 5C show that the defects treated with rASCs-laden GelMA/ PAMAM-MA hydrogels showed good neotissue filling with regular, surfaces and were indistinguishable from the surrounding tissues. The defects filled with rASCsladen GelMA hydrogels displayed moderate new tissue coverage and surface regularity. The blank control group showed an irregular articular surface in the defect area, 


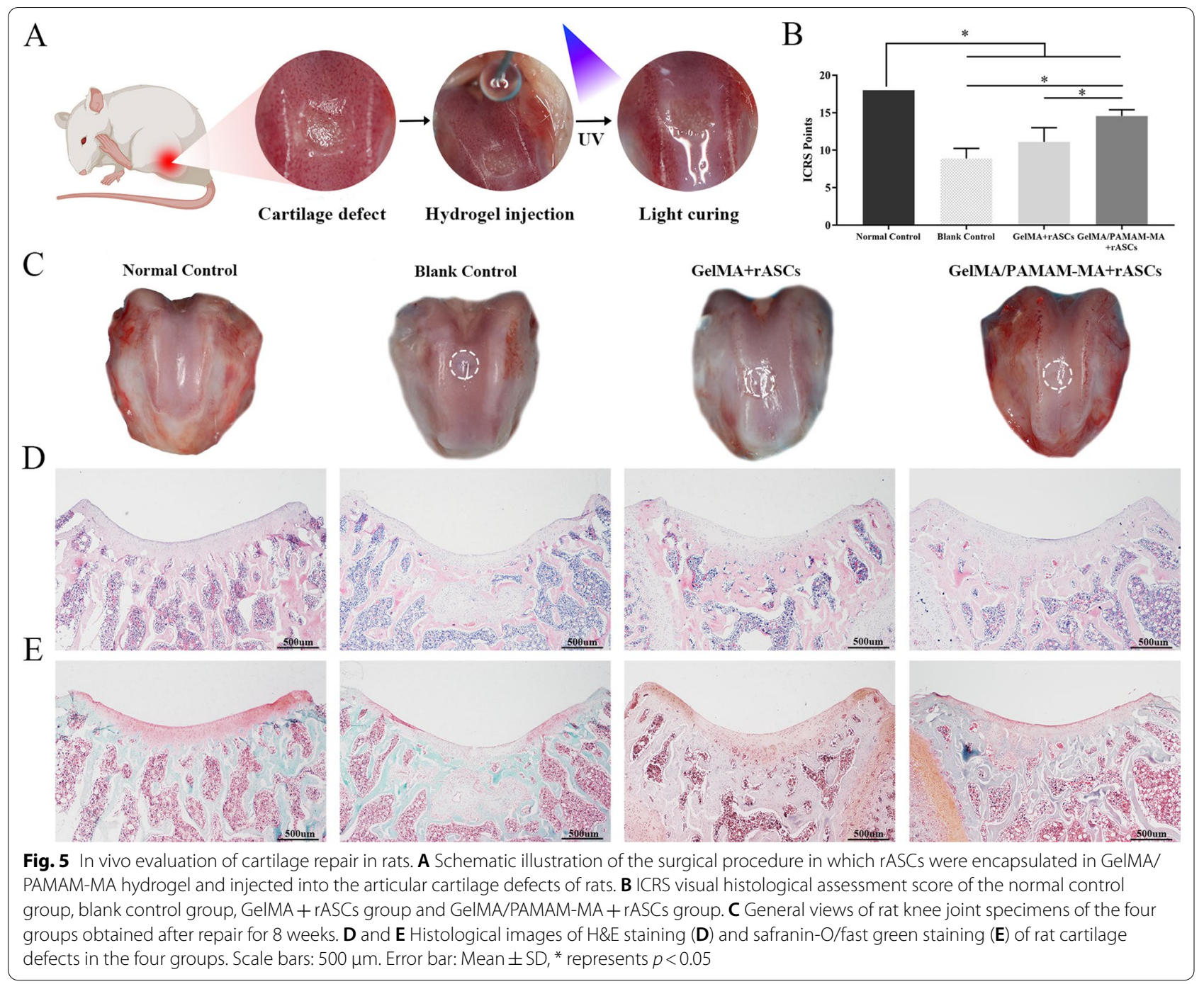

indicating insufficient filling and inadequate repair of the cartilage tissue.

$H \& E$ staining was performed to examine cartilage regeneration in the defect areas (Fig. 5D). When comparing the two groups of hydrogels, we found that the GelMA/PAMAM-MA hydrogel yielded better regeneration than the GelMA group since the defect almost disappeared, while a clear boundary between the neotissue and the adjacent natural cartilage could be observed in the GelMA hydrogel group. For the blank control group, the defect area was filled with fibrous tissues and a concave surface below the height of normal tissue could be observed, which confirms that cartilage was difficult to self-heal. Furthermore, safranin-O/ fast green staining was used to assess the quality of the repaired cartilage (Fig. 5E). Representative images show that the GAGs content was deposited in the neotissue in the GelMA/PAMAM-MA + rASCs group, while no typical GAGs content was found in the defect areas of the other groups. The results of the ICRS visual histological assessment score showed that the GelMA/ PAMAM-MA hydrogel group displayed significantly higher scores than did both the GelMA hydrogel group and blank control group (Fig. 5B).

In addition, we carried out IHC staining of cartilageassociated protein type II collagen and SOX9. The results of Col-II staining showed that more positively stained areas could be detected in the cartilage defect repair area of the GelMA/PAMAM-MA + rASCs group than in the GelMA+rASCs group, and the cartilage defect area of the blank control group was essentially the result of negative staining (Fig. 6A, C). Similarly, the percentage of SOX9-positive cells in the GelMA/PAMAM-MA + rASCs group was significantly higher than that in both the GelMA + rASCs group and the blank control group (Fig. 6B, D). Taken 


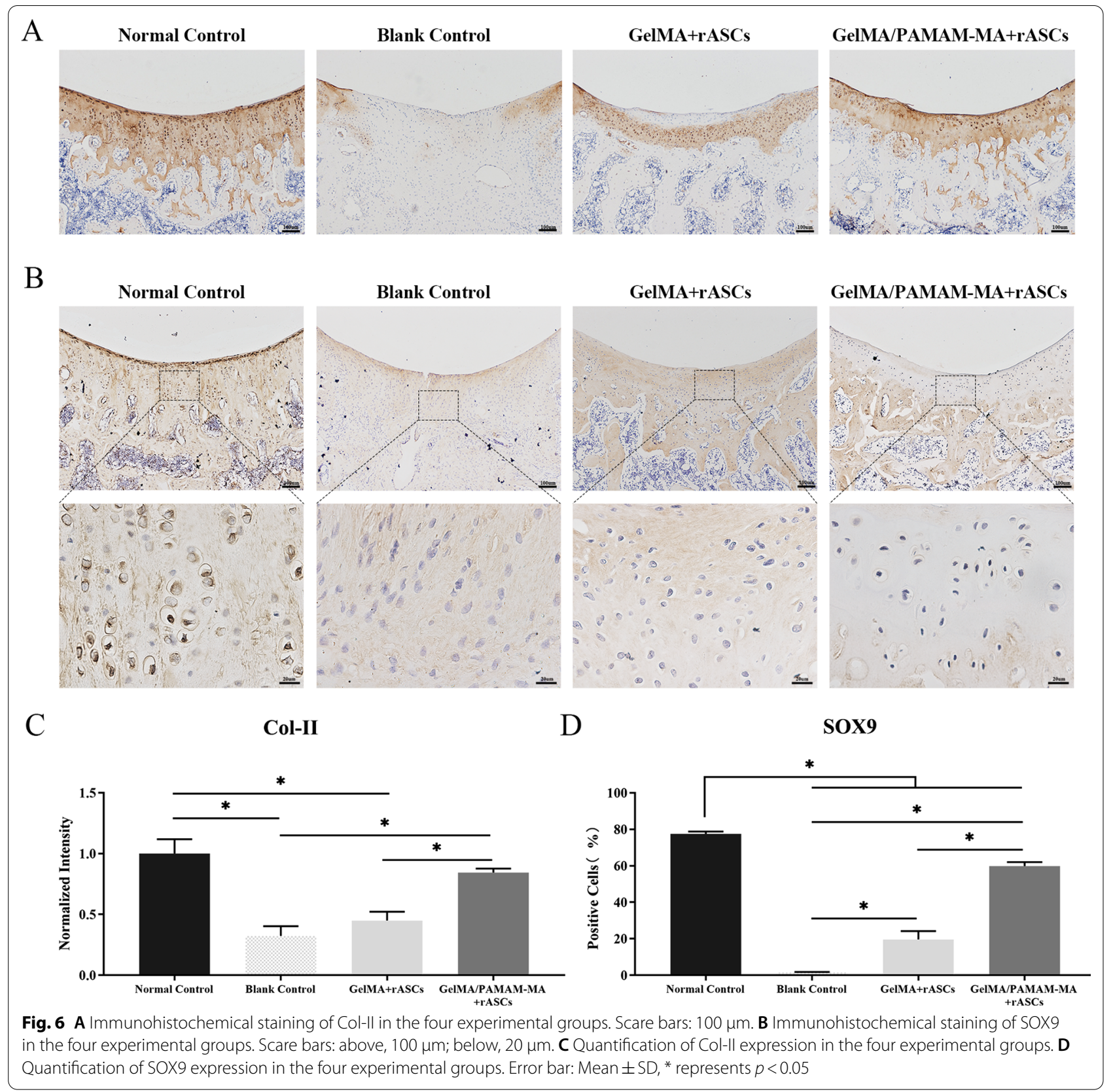

together, the above results indicated that, compared with the other treatments, the GelMA/PAMAMMA hydrogel presented better in vivo cartilage repair ability.

\section{Discussion}

In this study, we developed an injectable photocrosslinked hydrogel for ASCs delivery by combining GelMA with PAMAM-MA. The stability and mechanical properties of the photocrosslinked hydrogel were reinforced by incorporating PAMAM-MA into the GelMA hydrogel without damaging the biocompatibility. Moreover, the efficiency of the in vitro chondrogenic induction of the differentiation ability of the GelMA/PAMAMMA hydrogel significantly improved compared with that of the GelMA hydrogel group. In addition, we proved that, compared with the other hydrogels, the GelMA/ PAMAM-MA hydrogel has better efficiency in improving the in vivo cartilage regeneration.

Owing to their abundance, ready accessibility, nonimmunogenic and anti-inflammatory properties, ASCs are considered ideal sources of stem cells [38]. Some 
researchers have developed the preliminary clinical trials of the intra-articular injection of ASCs into osteoarthritis patients for cartilage regeneration therapy [39, 40]. However, there were some deficiencies in stem cell injection therapy, such as the adverse effects caused by easy diffusion and the inaccurate delivery of stem cells $[12,41]$. Therefore, to accurately implant cells into the targeted cartilage defect areas and effectively induce the chondrogenic differentiation of ASCs, it is necessary to develop the cell delivery scaffolds with good biocompatibility, feasibility and chondrogenic induction ability [42]. In this regard, we have developed an injectable photocrosslinked hydrogel for ASCs delivery. This GelMA/ PAMAM-MA hydrogel has been improved to have exert biocompatibility and cell affinity. Moreover, the incorporation of PAMAM-MA with GelMA not only improved the stability and mechanical properties of the hydrogel but also improved the chondrogenic differentiation ability of the encapsulated rASCs, which was verified by a series of physiochemical characterization tests as well as by in vitro and in vivo chondrogenesis studies.

The physicochemical properties of stem cell-loaded hydrogels are crucial in regulating cell viability and cell fate $[43,44]$. The photocrosslinked GelMA hydrogel has good biocompatibility, cell affinity and plasticity, while its poor mechanical properties hinder its cartilage differentiation-inducing abilities. Therefore, we incorporated PAMAM-MA into the single component GelMA hydrogel to optimize the physiochemical abilities. By detecting the internal structure of hydrogels under two conditions of dry and wet conditions, we found that the addition of PAMAM-MA can reduce the pore sizes of the hydrogel and make the structure more compact and thoroughly distributed. In addition, the stability of the hydrogel was also improved, as the swelling ratio and the in vitro degradation rate of the GelMA/PAMAM-MA hydrogel greatly decreased compared with those of the GelMA hydrogel. These improvements enabled hydrogels to continuously immobilize stem cells to targeted locations, which is essential for effective cartilage defect repair. Moreover, the mechanical properties of hydrogels have been proven to play important roles in regulating stem cell fate, especially cell differentiation [45]. Previous studies have shown that stem cells tend to differentiate into adipose cells in a softer matrix, while in a stiffer matrix, they are preferred for chondrogenic and osteogenic differentiation [46]. Directional stem cell differentiation induction could be achieved by adjusting hydrogels to simulate the stiffness of the ECM of targeted cells [47]. Some researchers have modulated the stiffness of the photocrosslinked hydrogels by altering the degree of methacrylation and stiffer hydrogels can better induce the chondrogenesis of human ASCs [48]. However, the increase in the degree of methacrylation would damage cell vitalities. In this research, the mechanical properties of this composite hydrogel increased significantly with the addition of PAMAM-MA. The compressive modulus of hydrogels increased from $2.56 \pm 0.26 \mathrm{kPa}$ to $8.06 \pm 1.39 \mathrm{kPa}$. Moreover, the cell viability of the encapsulated rASCs was not hindered.

After encapsulating rASCs into two groups of GelMA/ PAMAM-MA hydrogels for 3D culture, we detected the biocompatibility of the hydrogels. The live/dead cell staining results showed that cells cultured in the two hydrogels had good vitality at all time points, and the addition of PAMAM-MA had a negligible effect on the viability of rASCs. In addition, we injected the rASCsloaded GelMA/PAMAM-MA hydrogel into rat articular cartilage defects. These rASCs were transfected with GFP-labeled adenovirus. At 1 week, 2 weeks and 4 weeks after implantation, these rASCs could still be observed in the cartilage defect areas. Overall, the good biocompatibility of the photocrosslinked hydrogel was not affected by the addition of PAMAM-MA. Moreover, we analyzed the cell morphology of the encapsulated rASCs in the two groups of hydrogels. Cells loaded in the GelMA/ PAMAM-MA hydrogel presented reduced spreading areas and a more spherical shape, similar to that of chondrocytes, which might be beneficial to chondrogenic differentiation of rASCs [49].

The physiochemical properties of hydrogels have been proven to play important roles in regulating stem cell fate, especially cell differentiation [19]. In view of this, we encapsulated rASCs into two groups of GelMA/ PAMAM-MA hydrogels to determine their in vitro chondrogenic differentiation induction abilities. The expression of chondrogenic-related genes in rASCs was detected after 7 days of 3D culture. The expression levels of SOX9, ACAN and Col-II significantly increased in the GelMA/PAMAM-MA hydrogel, which indicated that the changes in physical properties of the GelMA/ PAMAM-MA hydrogel induced by the addition of PAMAM-MA could directly affect the early chondrogenic differentiation abilities of rASCs. The expression of the hypertrophic chondrocyte-related gene Col-X showed no significant difference between the two groups, which was frequently mentioned as the main disadvantage of utilizing stem cells for cartilage repair [4]. Furthermore, the spatial distribution of GAGs and the protein expression level of SOX9 in the two groups of hydrogels further demonstrated that rASCs loaded in the GelMA/PAMAM-MA hydrogel had a better effect on chondrogenic differentiation in vitro. In general, compared with the GelMA hydrogel, the GelMA/PAMAMMA hydrogel was proven to have better chondrogenic differentiation-inducing ability, which can be attributed 
to the optimization of the physiochemical and mechanical properties of the GelMA/PAMAM-MA hydrogel. However, we did not discuss in depth how the physical properties of hydrogels regulate the differentiation of stem cells, which was also a deficiency of this study. In our follow-up study, we can further conduct research on the regulation of stem cell differentiation by physical and mechanical signal transduction and provide a more reliable basis for the optimal design of stem cell delivery scaffold materials.

After the rASCs-loaded hydrogels were injected into rat articular cartilage defects for 8 weeks, we tested the repair effects by gross morphological examining, histological staining and IHC staining. Newly formed cartilage tissue filling and negligible boundaries were observed in the GelMA/PAMAM-MA hydrogel-filled cartilage defect areas, while the GelMA hydrogel group and blank control group showed irregular surfaces and clear boundaries between the neotissue and the adjacent natural cartilage. According to the following histological staining results of $\mathrm{H} \& \mathrm{E}$ staining, safranin-O/fast green staining and IHC staining (Col-II and SOX9), neocartilage tissue was formed in the GelMA/PAMAM-MA group; this tissue was tightly integrated with surrounding cartilage tissues. A mixture of cartilage and fibrous tissue was produced in the GelMA group and only fibrous-like tissue formed in the blank control group. In summary, the GelMA/ PAMAM-MA hydrogel loaded with rASCs had a better cartilage repair effect than the GelMA hydrogel, which was consistent with the effects of our in vitro chondrogenic induction differentiation analysis.

\section{Conclusions}

In this study, by combining GelMA and PAMAM-MA, we constructed an injectable photocrosslinked hydrogel to deliver rASCs for cartilage defect repair. The addition of PAMAM-MA significantly improved the uniformity of the internal network and the stability and mechanical properties of the GelMA hydrogel. Moreover, the biocompatibility of GelMA/PAMAM-MA hydrogels was not hindered. The capacity of the hybrid hydrogels to induce chondrogenic differentiation of rASCs in vitro was also significantly enhanced with the optimization of the physiochemical properties of the hydrogels. Finally, we verified the excellent ability of GelMA/PAMAM-MA hydrogels to promote cartilage regeneration through in vivo cartilage defect repair experiments. This injectable hybrid hydrogel is promising for application as a stem cell delivery tissue engineering scaffold and for eventually implementing a successful stem cell-based tissue engineering cartilage regeneration strategy.

\section{Abbreviations}

GeIMA: Gelatin methacrylate; MA: Methacrylic anhydride; PAMAM-MA: Methacrylic anhydride-modified poly(amidoamine); PAMAM: Poly(amidoamine); ASCs: Adipose-derived stromal/stem cells; 3D: Three-dimensional; PEG-LADA: Poly(lactic acid)-b-poly(ethylene glycol)-b-poly(lactic acid) with acrylate end-groups; PBS: Phosphate-buffered saline; FTIR: Fourier transform infrared spectroscopy; Irgacure 2959: 2-Hydroxy-1(4-(hydroxyethoxy)pheny)-2-methyl1-propanone; UV: Ultraviolet; SEM: Scanning electron microscopy; OCT: Optimal cutting temperature compound; Wi: Initial weight; Wd: Dry weight; rASCs: Rat adipose-derived stromal/stem cells; DMEM: Dulbecco's modified Eagle's medium; FCM: Flow cytometry; PDMS: Polydimethylsiloxane; DAPI: 4',6-Diamidino-2-phenylindole; PFA: Paraformaldehyde; qPCR: Quantitative real-time polymerase chain reaction; SOX9: Sex determining region Y-box transcription factor 9; ACAN: Aggrecan; Col-II: Type II collagen; Col-X: Type X collagen; GAPDH: Glyceraldehyde-3-phosphate dehydrogenase; cDNA: Complementary DNA; ECM: Extracellular matrix; IF: Immunofluorescence; GFP: Green fluorescent protein; EDTA: Ethylenediaminetetraacetic acid; H\&E: Hematoxylin and eosin; ICRS: International cartilage repair society; IHC: Immunohistochemical; DAB: 3,3'-diaminobenzidine; SD: Standard deviation.

\section{Supplementary Information}

The online version contains supplementary material available at https://doi. org/10.1186/s13287-022-02705-6.

Additional file 1. Supplementary information, Table S1. Supplementary information, Fig. S1 and Supplementary information, Fig. S2.

\section{Acknowledgements}

Not applicable.

\section{Authors' contributions}

$F Y L, J X, S Y$ and $P J$ were responsible for the conception and design of the study; FYL, MXR, PH and LW performed the collection, assembly of the data; $X W, Y Z L, M X R$ carried out the analysis and interpretation of the data; FYL, XW were major contributors in writing the manuscript; PJ, SY and JX were responsible for the critical revision of the article for important intellectual content. All authors read and approved the final manuscript.

\section{Funding}

This work was supported by the National Natural Science Foundation of China (Grant Nos. 82171010, 82001103, 82071115, 31871464, 81800999) for the design of the experiment and the purchase of the experimental reagents, the Natural Science Foundation of Chongqing, China (Grant Nos. cstc2021jcyj-jqX0028, cstc2019jcyj-msxmX0366, cstc2019jcyj-bshX0005, cstc2019jcyj-msxmX0150) for collection, analysis, and interpretation of data, and the Chongqing Medical University Chongqing Postgraduate Tutor Team Construction Project (dstd201806) for writing the manuscript.

\section{Availability of data and materials}

The datasets used and analyzed during the current study are available from the corresponding author on reasonable request.

\section{Declarations}

\section{Ethics approval and consent to participate}

The animal experiments in this study were carried out in compliance with the Animal Welfare Act and in the standard surgical procedures that were approved by the Ethics Committee of Stomatological hospital of Chongqing Medical University [CQHS-REC-2020(LSNo.46)].

\section{Consent for publication}

Not applicable.

\section{Competing interests}

The authors declare that they have no competing interests. 


\section{Author details}

${ }^{1}$ College of Stomatology, Chongqing Medical University, Chongqing, China. ${ }^{2}$ Chongqing Key Laboratory of Oral Diseases and Biomedical Sciences, Chongqing, China. ${ }^{3}$ Chongqing Municipal Key Laboratory of Oral Biomedical Engineering of Higher Education, Chongqing, China.

Received: 22 September 2021 Accepted: 16 December 2021 Published online: 24 January 2022

\section{References}

1. Buckwalter JA, Mankin HJ. Articular cartilage repair and transplantation. Arthritis Rheum. 1998;41(8):1331-42.

2. Medvedeva EV, Grebenik EA, Gornostaeva SN, Telpuhov VI, Lychagin AV, Timashev PS, et al. Repair of damaged articular cartilage: current approaches and future directions. Int J Mol Sci. 2018;19(8):2366.

3. Vinatier C, Guicheux J. Cartilage tissue engineering: from biomaterials and stem cells to osteoarthritis treatments. Ann Phys Rehabil Med. 2016:59(3):139-44.

4. Armiento AR, Alini M, Stoddart MJ. Articular fibrocartilage-Why does hyaline cartilage fail to repair? Adv Drug Deliv Rev. 2019;146:289-305.

5. Tuan RS, Chen AF, Klatt BA. Cartilage regeneration. J Am Acad Orthop Surg. 2013;21(5):303-11.

6. Kwon H, Brown WE, Lee CA, Wang D, Paschos N, Hu JC, et al. Surgical and tissue engineering strategies for articular cartilage and meniscus repair. Nat Rev Rheumatol. 2019;15(9):550-70.

7. Bhattacharjee M, Coburn J, Centola M, Murab S, Barbero A, Kaplan DL, et al. Tissue engineering strategies to study cartilage development, degeneration and regeneration. Adv Drug Deliv Rev. 2015;84:107-22.

8. Murphy MP, Koepke LS, Lopez MT, Tong X, Ambrosi TH, Gulati GS, et al Articular cartilage regeneration by activated skeletal stem cells. Nat Med. 2020;26(10):1583-92.

9. Dai R, Wang Z, Samanipour R, Koo Kl, Kim K. Adipose-derived stem cells for tissue engineering and regenerative medicine applications. Stem Cells Int. 2016;2016:6737345.

10. Bacakova L, Zarubova J, Travnickova M, Musilkova J, Pajorova J, Slepicka P, et al. Stem cells: their source, potency and use in regenerative therapies with focus on adipose-derived stem cells - a review. Biotechnol Adv. 2018;36(4):1111-26.

11. Fraser JK, Wulur I, Alfonso Z, Hedrick MH. Fat tissue: an underappreciated source of stem cells for biotechnology. Trends Biotechnol. 2006;24(4):150-4.

12. Glotzbach JP, Wong VW, Levi B, Longaker MT, Gurtner GC. Delivery strategies for stem cell-based therapy. J Healthc Eng. 2012;3(1):1-20.

13. García-Bernal D, García-Arranz M, Yáñez RM, Hervás-Salcedo R, Cortés A, Fernández-García M, et al. The current status of mesenchymal stromal cells: controversies, unresolved issues and some promising solutions to improve their therapeutic efficacy. Front Cell Dev biol. 2021;9:650664.

14. Rustad KC, Wong WW, Sorkin M, Glotzbach JP, Major MR, Rajadas J, et al. Enhancement of mesenchymal stem cell angiogenic capacity and stemness by a biomimetic hydrogel scaffold. Biomaterials. 2012;33(1):80-90

15. González-Cruz RD, Fonseca VC, Darling EM. Cellular mechanical properties reflect the differentiation potential of adipose-derived mesenchymal stem cells. Proc Natl Acad Sci USA. 2012;109(24):E1523-9.

16. Hwang NS, Varghese S, Elisseeff J. Controlled differentiation of stem cells. Adv Drug Deliv Rev. 2008;60(2):199-214.

17. Naahidi S, Jafari M, Logan M, Wang Y, Yuan Y, Bae H, et al. Biocompatibility of hydrogel-based scaffolds for tissue engineering applications. Biotechnol Adv. 2017;35(5):530-44.

18. Wang Y. Programmable hydrogels. Biomaterials. 2018;178:663-80.

19. Huang Q, Zou Y, Arno MC, Chen S, Wang T, Gao J, et al. Hydrogel scaffolds for differentiation of adipose-derived stem cells. Chem Soc Rev. 2017;46(20):6255-75.

20. Pascual-Garrido C, Rodriguez-Fontan F, Aisenbrey EA, Payne KA, Chahla J, Goodrich LR, et al. Current and novel injectable hydrogels to treat focal chondral lesions: Properties and applicability. J Orthop Res. 2018;36(1):64-75.
21. Wu J, Chen Q, Deng C, Xu B, Zhang Z, Yang Y, et al. Exquisite design of injectable hydrogels in cartilage repair. Theranostics. 2020;10(21):9843-64.

22. Annabi N, Tamayol A, Uquillas JA, Akbari M, Bertassoni LE, Cha C, et al. 25th anniversary article: Rational design and applications of hydrogels in regenerative medicine. Adv Mater. 2014;26(1):85-123.

23. Xiao S, Zhao T, Wang J, Wang C, Du J, Ying L, et al. Gelatin methacrylate (GelMA)-based hydrogels for cell transplantation: an effective strategy for tissue engineering. Stem Cell Rev Rep. 2019;15(5):664-79.

24. Klotz BJ, Gawlitta D, Rosenberg A, Malda J, Melchels FPW. Gelatin-methacryloyl hydrogels: towards biofabrication-based tissue repair. Trends Biotechnol. 2016;34(5):394-407.

25. Sun M, Sun X, Wang Z, Guo S, Yu G, Yang H. Synthesis and properties of gelatin methacryloyl (GelMA) hydrogels and their recent applications in load-bearing tissue. Polymers (Basel). 2018;10(11):1290.

26. Yue K, Trujillo-de Santiago G, Alvarez MM, Tamayol A, Annabi N, Khademhosseini A. Synthesis, properties, and biomedical applications of gelatin methacryloyl (GelMA) hydrogels. Biomaterials. 2015;73:254-71.

27. Svenson S, Tomalia DA. Dendrimers in biomedical applications-reflections on the field. Adv Drug Deliv Rev. 2005;57(15):2106-29.

28. Zhou Y, Huang W, Liu J, Zhu X, Yan D. Self-assembly of hyperbranched polymers and its biomedical applications. Adv Mater. 2010;22(41):4567-90

29. Taghavi Pourianazar N, Mutlu P, Gunduz U. Bioapplications of poly(amidoamine) (PAMAM) dendrimers in nanomedicine. J Nanopart Res. 2014;16(4):2342.

30. Araújo RV, Santos SDS, Igne Ferreira E, Giarolla J. New advances in general biomedical applications of PAMAM dendrimers. Molecules. 2018;23(11):2849.

31. Jenjob R, Phakkeeree T, Crespy D. Core-shell particles for drugdelivery, bioimaging, sensing, and tissue engineering. Biomater Sci. 2020;8(10):2756-70.

32. Maji S, Agarwal T, Maiti TK. PAMAM (generation 4) incorporated gelatin 3D matrix as an improved dermal substitute for skin tissue engineering. Colloids Surf B Biointerfaces. 2017;155:128-34.

33. Gorain B, Tekade M, Kesharwani P, Iyer AK, Kalia K, Tekade RK. The use of nanoscaffolds and dendrimers in tissue engineering. Drug Discov Today. 2017;22(4):652-64.

34. Wang $Y$, Zhao Q, Zhang $H$, Yang S, Jia X. A novel poly(amido amine)-dendrimer-based hydrogel as a mimic for the extracellular matrix. Adv Mater. 2014;26(24):4163-7.

35. Loessner D, Meinert C, Kaemmerer E, Martine LC, Yue K, Levett PA, et al. Functionalization, preparation and use of cell-laden gelatin methacryloyl-based hydrogels as modular tissue culture platforms. Nat Protoc. 2016;11(4):727-46.

36. Megaloikonomos PD, Panagopoulos GN, Bami M, Igoumenou VG, Dimopoulos L, Milonaki A, et al. Harvesting, isolation and differentiation of rat adipose-derived stem cells. Curr Pharm Biotechnol. 2018;19(1):19-29.

37. Mainil-Varlet P, Aigner T, Brittberg M, Bullough P, Hollander A, Hunziker E, et al. Histological assessment of cartilage repair: a report by the histology endpoint committee of the international cartilage repair society (ICRS). J Bone Joint Surg Am. 2003;85(suppl_2):45-57.

38. Makris EA, Gomoll AH, Malizos KN, Hu JC, Athanasiou KA. Repair and tissue engineering techniques for articular cartilage. Nat Rev Rheumatol. 2015;11(1):21-34.

39. Lu L, Dai C, Zhang Z, Du H, Li S, Ye P, et al. Treatment of knee osteoarthritis with intra-articular injection of autologous adipose-derived mesenchymal progenitor cells: a prospective, randomized, double-blind, activecontrolled, phase Ilb clinical trial. Stem Cell Res Ther. 2019;10(1):143.

40. Koh YG, Choi YJ, Kwon SK, Kim YS, Yeo JE. Clinical results and secondlook arthroscopic findings after treatment with adipose-derived stem cells for knee osteoarthritis. Knee Surg Sports Traumatol Arthrosc 2015;23(5):1308-16.

41. Wagner J, Kean T, Young R, Dennis JE, Caplan Al. Optimizing mesenchymal stem cell-based therapeutics. Curr Opin Biotechnol. 2009;20(5):531-6

42. Wei W, Ma Y, Yao X, Zhou W, Wang X, Li C, et al. Advanced hydrogels for the repair of cartilage defects and regeneration. Bioact Mater. 2021;6(4):998-1011.

43. Lutolf MP, Gilbert PM, Blau HM. Designing materials to direct stem-cell fate. Nature. 2009;462(7272):433-41. 
44. Tsou YH, Khoneisser J, Huang PC, Xu X. Hydrogel as a bioactive material to regulate stem cell fate. Bioact Mater. 2016;1 (1):39-55.

45. Xu B, Ye J, Yuan FZ, Zhang JY, Chen YR, Fan BS, et al. Advances of stem cell-laden hydrogels with biomimetic microenvironment for osteochondral repair. Front Bioeng Biotechnol. 2020;8:247.

46. Lv H, Li L, Sun M, Zhang Y, Chen L, Rong Y, et al. Mechanism of regulation of stem cell differentiation by matrix stiffness. Stem Cell Res Ther. 2015;6(1):103

47. Ma Y, Lin M, Huang G, Li Y, Wang S, Bai G, et al. 3D spatiotemporal mechanical microenvironment: a hydrogel-based platform for guiding stem cell fate. Adv Mater. 2018;30(49):e1705911.

48. Teong B, Wu SC, Chang CM, Chen JW, Chen HT, Chen CH, et al. The stiffness of a crosslinked hyaluronan hydrogel affects its chondroinduction activity on hADSCs. J Biomed Mater Res B Appl Biomater. 2018;106(2):808-16.

49. Mathieu PS, Loboa EG. Cytoskeletal and focal adhesion influences on mesenchymal stem cell shape, mechanical properties, and differentiation down osteogenic, adipogenic, and chondrogenic pathways. Tissue Eng Part B Rev. 2012;18(6):436-44.

\section{Publisher's Note}

Springer Nature remains neutral with regard to jurisdictional claims in published maps and institutional affiliations.

- fast, convenient online submission

- thorough peer review by experienced researchers in your field

- rapid publication on acceptance

- support for research data, including large and complex data types

- gold Open Access which fosters wider collaboration and increased citations

- maximum visibility for your research: over 100M website views per year

At BMC, research is always in progress.

Learn more biomedcentral.com/submissions 